\title{
AN ITERATIVE METHOD FOR APPROXIMATING LTI SYSTEMS USING SUBBANDS
}

\author{
Damián Marelli $i^{\dagger}$ Minyue Fu $u^{\dagger}$, Peter Balazs ${ }^{\ddagger}$ and Piotr Majdak $k^{\ddagger}$ \\ ${ }^{\dagger}$ School of Electrical Engineering and Computer Science, University of Newcastle, Australia. \\ ${ }^{\ddagger}$ Acoustics Research Institute, Austrian Academy of Science, Austria.
}

\begin{abstract}
A linear system can be approximated in the time-frequency domain by the composition of an analysis filterbank, a transfer matrix (subband model) and a synthesis filterbank, a method known as subband technique. In this paper we propose an iterative method to jointly optimize the subband model, analysis and synthesis filterbanks. To this end we propose a minimization criterion which we solve using the so-called alternating least-squares method. As a possible application we consider the implementation of the so-called head related transfer functions which are used in virtual acoustics. Simulation results suggest that the subband technique, optimized using the proposed method proposed method, is a promising approach.
\end{abstract}

keywords: Subband filters, Time-frequency analysis, Headrelated transfer functions.

\section{INTRODUCTION}

The subband technique represents a linear system in the timefrequency domain. More precisely, the system is replaced by the composition of an analysis filterbank, followed by a (usually diagonal) transfer matrix (called the subband model) and a synthesis filterbank. This approach can be used for system approximation [1], system identification [2], adaptive filtering [3], channel equalization [4], etc., with the advantage of having a higher numerical efficiency. However, the analysis of this technique and optimal setup are not trivial.

The approximation of Hilbert-Schmidt operators (i.e., a kind of linear time-variant (LTV) system) by the so-called Gabor multipliers (i.e., a diagonal subband model without memory) has been studied in [5, 6], where, for given choices of analysis and synthesis filterbanks, the subband model is chosen to minimize the Hilbert-Schmidt norm. The case of linear time-invariant (LTI) systems, was studied in [1], where the optimal subband model was chosen to minimize the power of the output error signal, assuming a white input signal.

In this paper we proceed a step further from [1]. More precisely, we propose an iterative method to jointly optimize

This work was partially developed at the Faculty of Mathematics, NuHAG, University of Vienna, with financial support from the EU Marie Curie fellowship MEIF-CT-2006-023728. the choices of the subband model, analysis and synthesis filterbanks, when the quality of the approximation is measured by the power of the output error signal, assuming that the input signal has an arbitrary but known power spectrum. To this end, we consider the so-called alternating least-squares (ALS) algorithm [7]. which consists of cyclic iterations where, at each step, two of the three elements to be optimized are fixed, and the third is optimized using linear least-squares (LLS).

The time-domain implementation of an LTI system is computationally inefficient, and it is often prohibitive for some applications, e.g., real-time audio applications where impulse responses are in the order of several hundreds. A computationally efficient alternative implements the system in the frequency domain; however, this approach is not suitable for real-time applications, because it requires the block processing of the "whole history" of the involved signals. To address this issue, and for the case of systems of finite impulse response (FIR) type, the so-called overlap-save and overlap-add methods (OS/A) have been proposed [8]. Both methods permit accommodating a trade-off between computational complexity and latency (i.e., time-delay). Simulation results show that a subband scheme, optimized using the algorithm described above, offers a better trade-off than that of the OS/A methods, provided a tolerance on the implementation error is allowed. (Notice that an implementation error is unavoidably introduced when using OS/A methods to implement a system of infinite impulse response (IIR) type.)

In order to illustrate this point, we consider the implementation of the so-called head-related transfer functions (HRTFs), which find applications in the so-called binaural virtual acoustics synthesis [9]. This technique consists of modifying a sound source signal, so as to give the listener the sensation that it is located in space. This is done by filtering the signal from the source using an HRTF, which describes the filtering effects of the listener's morphology (i.e. pinna, head, torso, etc.) The HRTFs are given separately for the left and right ears, and their associated head related impulse responses (HRIR) are functions of the source location relative to the listener head's position. In a virtual acoustics application, a pair of filters needs to be computed for every sound source location, which is often computationally unaffordable in real-time applications.

Throughout the paper we use the following notation: com- 
plex sequences (indexed by the integers $\mathbb{Z}$ ) are denoted using non-bold lowercase letters (e.g., a). Vectors and matrices whose elements are complex sequences are denoted by lowercase bold letters (e.g., a) and uppercase bold letters (e.g., A), respectively. Complex-valued vectors and matrices are denoted using underlined letters (e.g., $\underline{\mathbf{a}}$ and $\underline{\mathbf{A}}$ ). An LTI system (or filter) $g(q)$ is denoted as a function of the forward shift operator $q$ (i.e., $q x(t)=x(t+1)$ ), and its impulse response is denoted by $g(t)$. Finally, we say that an LTI system $g(q)$ is FIR, with tap size $l_{g}$ and $d_{g}$ non-causal taps, if $g(t)=0$ for all $t \notin\left\{-d_{g}, \cdots, l_{g}-d_{g}-1\right\}$.

The proofs of results are not included in the paper and will be included in a journal version.

\section{SYSTEM APPROXIMATION USING SUBBANDS}

The subband approximation scheme is shown in Fig. 1. The linear system $g(q)$ is approximated by splitting the input signal $x(t)$ into $M$ subbands using the array of filters $\mathbf{h}(q)=$ $\left[h_{1}(q), \cdots, h_{M}(q)\right]^{T}$, followed by a downsampling operation of factor $D$ (i.e., one out of $D$ samples is kept). In this way, the subband vector signal $\boldsymbol{\xi}(t)$ is generated. The subband model is an $M \times M$ transfer matrix $\boldsymbol{\Gamma}(q)$ whose output is denoted by $\hat{\boldsymbol{\theta}}(t)$. Finally, the output signal $\hat{y}(t)$ is generated by upsampling $\hat{\boldsymbol{\theta}}(t)$ by a factor of $D$ (i.e., $D-1$ zero valued samples are added between every two samples), then filtering each component using the array of filters $\mathbf{f}^{*}(q)=$ $\left[f_{1}^{*}(q), \cdots, f_{M}^{*}(q)\right]$, and adding together all the resulting signals.

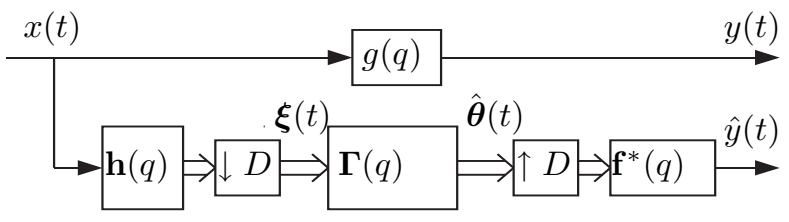

Fig. 1. System approximation in the time-frequency domain.

We assume that the system $g(q)$ is an IIR system. Also, the filters in the arrays $\mathbf{h}(q)$ and $\mathbf{f}(q)$ are causal and FIR with tap sizes $l_{\mathbf{h}}$ and $l_{\mathbf{f}}$, respectively. Finally, the subband model $\boldsymbol{\Gamma}(q)$ is diagonal with FIR filters of tap size $l_{\boldsymbol{\Gamma}}$ and $d_{\boldsymbol{\Gamma}}$ noncausal taps, on each diagonal entry.

Comparison with OS/A methods: These methods permit the implementation of an FIR system, whose tap size we denote by $l_{g}$. They consist of an iterative procedure in which, at each iteration, a block of $l_{g}+L-1$ samples is processed in the frequency domain. Then, $L$ samples are skipped, and the next iteration is processed [8]. The computational cost and latency of the OS/A and subband methods are given in Table 1, where we assume that the filterbanks are of Gabor type (i.e., there exists $h_{0}(t)$ such that, for all $m \in\{1, \cdots, M\}$ and all $t \in \mathbb{Z}, h_{m}(t)=e^{j \frac{2 \pi}{M}(m-1) t} h_{0}(t)$, and a similar property for

\begin{tabular}{|c|c|c|}
\hline & CC [mult./sample] & Latency [samples] \\
\hline OS/A & $\frac{l_{g}+L-1}{L} \log _{2} 2\left(l_{g}+L-1\right)$ & $L-1$ \\
\hline SB & $\frac{M}{D}\left(l_{\Gamma}+\frac{l_{\mathbf{h}}+l_{\mathrm{f}}}{M}+\log _{2} M\right)$ & $d_{\Gamma} D+l_{\mathbf{f}}-1$ \\
\hline
\end{tabular}

Table 1. Computational cost (CC) and latency for the OS/A and subband (SB) methods.

$\mathbf{f}(t)$ ), for which a numerically efficient algorithm exists [10]. We also assume that a $k$-point FFT can be implemented with $\frac{k}{2} \log _{2} k$ multiplications.

\section{PROPOSED APPROXIMATION CRITERION}

The goal is to find the filters $\mathbf{h}(q), \mathbf{f}(q)$ and the subband model $\boldsymbol{\Gamma}(q)$, for given values of $l_{\mathbf{h}}, l_{\mathbf{f}}, l_{\boldsymbol{\Gamma}}, d_{\boldsymbol{\Gamma}}, M$ and $D$, that minimize the power of the error signal $\tilde{y}(t)=y(t)-\hat{y}(t)$, when $x(t)$ has a given auto-correlation function $r_{x}(t)$. In this section we express this problem as a minimization problem. To this end, we transform the setting in Fig. 1 using the so-called polyphase representation [11].

\subsection{Polyphase Representation}

Let $x(t)$ be a scalar random process. The polyphase representation of $x(t)$ is the vector random process $\mathbf{x}(t)$ satisfying

$$
[\mathbf{x}]_{d}=\phi_{1-d}^{D} x \text { for each } d \in\{1, \cdots, D\},
$$

where $[\mathbf{x}]_{d}$ denotes the $d$-th entry of $\mathbf{x}(t)$ and $\left(\phi_{e}^{D} x\right)(t)=$ $x(t D+e)$. Also, let $g(t)$ be the impulse response of an LTI system $g(q)$. The polyphase representation of $g(q)$ is the $D \times$ $D$ transfer matrix $\mathbf{G}(q)$ whose impulse response satisfies

$$
[\mathbf{G}]_{d, e}=\phi_{e-d}^{D} g \text { for each } d, e \in\{1, \cdots, D\}
$$

Also, the polyphase representation of an analysis filterbank with filters $\mathbf{h}(q)$ and downsampling factor $D$ is the $M \times D$ transfer matrix $\mathbf{H}(q)$ whose impulse response satisfies

$$
[\mathbf{H}]_{m, d}=\phi_{d-1}^{D} h_{m} ; m \in\{1, \cdots, M\}, d \in\{1, \cdots, D\}
$$

The polyphase representation of the synthesis filterbank $\mathbf{f}(q)$ with upsampling factor $D$ is the $D \times M$ transfer matrix $\mathbf{F}^{*}(q)$, where $\mathbf{F}(q)$ is defined as in (1), and $\mathbf{F}^{*}(q)=\mathbf{F}\left(q^{-1}\right)^{*}$, with $\mathbf{F}\left(q^{-1}\right)^{*}$ being the transpose conjugate of $\mathbf{F}\left(q^{-1}\right)$. If $\mathbf{h}(q)$ is of Gabor type, and $h_{0}(q)$ is a causal FIR filter with tap size $l_{\mathbf{h}}$, then its polyphase representation is given by [10]

$$
\mathbf{H}=\mathcal{W}_{M} \mathbf{L}_{2} \Lambda_{h_{0}} \mathbf{L}_{1}
$$

where $\mathcal{W}_{M} \in \mathbb{C}^{M \times M}$ is the DFT matrix, i.e., $\left[\mathcal{W}_{M}\right]_{k, l}=$ $M^{-1 / 2} e^{-j \frac{2 \pi}{M} k l}$ and

$$
\begin{aligned}
\mathbf{L}_{1}^{T} & =\left[\mathbf{I}_{D}, q^{-1} \mathbf{I}_{D}, \cdots, q^{-n_{D}+1} \mathbf{I}_{D}\right]_{:, 1: l_{\mathbf{h}}} \\
\mathbf{L}_{2} & =[\underbrace{\mathbf{I}_{M}, \mathbf{I}_{M} \cdots, \mathbf{I}_{M}}_{n_{M} \text { times }}]_{:, 1: l_{\mathbf{h}}} \\
\Lambda_{h_{0}} & =\operatorname{diag}\left\{h_{0}(0), \cdots, h_{0}\left(l_{\mathbf{h}}-1\right)\right\}
\end{aligned}
$$


with $n_{D}=\left\lceil\frac{l_{\mathbf{h}}}{D}\right\rceil, n_{M}=\left\lceil\frac{l_{\mathbf{h}}}{M}\right\rceil$ and $[\mathbf{A}]:, k: l$ denoting the matrix formed with the columns from $k$ to $l$ of $\mathbf{A}$. Also, for a vector $\underline{\mathbf{x}}, \operatorname{diag}\{\underline{\mathbf{x}}\}$ denotes the diagonal matrix with elements $[\mathbf{x}]_{i}$ in its main diagonal.

By using the polyphase representation, the scheme in Fig. 1 can be represented by the LTI system shown in Fig. 2 .

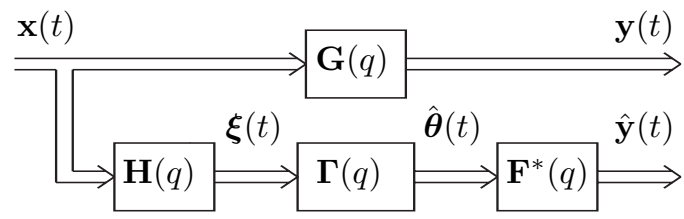

Fig. 2. Polyphase representation of the time-frequency system approximation scheme.

\subsection{Approximation as a Minimization Problem}

Let $\mathbf{G}(t)$ be the impulse response of the polyphase representation of an LTI system, let $x(t)$ and $y(t)$ denote its input and output, respectively, and let $\mathbf{x}(t)$ and $\mathbf{y}(t)$ denote their polyphase representations. Then, it is straightforward to verify that

$$
\mathbf{R}_{\mathbf{y}}=\mathbf{G} * \mathbf{R}_{\mathbf{x}} * \mathbf{G}^{*}
$$

where $*$ denotes convolution of matrix sequences and $\mathbf{R}_{\mathbf{v}}$ is the auto-correlation function of the vector random process $\mathbf{v}(t)$. It follows that

$$
S_{y}=\operatorname{Tr}\left\{\mathbf{G} * \mathbf{R}_{\mathbf{x}} * \mathbf{G}^{*}\right\}(0) S_{x}
$$

where $S_{v}$ is the power of the scalar random process $v(t)$, and for a $B \times B$ matrix sequence $\mathbf{X}(t), \operatorname{Tr}\{\mathbf{X}\}(t)=\sum_{i=1}^{B}[\mathbf{X}]_{i, i}(t)$. Hence, we define the following norm

$$
\|\mathbf{G}\|_{r_{x}}^{2}=S_{x}^{-1} \operatorname{Tr}\left\{\mathbf{G} * \mathbf{R}_{\mathbf{x}} * \mathbf{G}^{*}\right\}(0)
$$

which measures the power gain of the system when the input has auto-correlation $r_{x}(t)$.

Let $\left(\mathbb{C}^{B \times A}\right)^{\mathbb{Z}}$ be the space of sequences of complex $B \times A$ matrices indexed by $\mathbb{Z}$. Let $\mathcal{G} \subset\left(\mathbb{C}^{\mathbb{Z}}\right)^{M \times M}, \mathcal{H} \subset\left(\mathbb{C}^{\mathbb{Z}}\right)^{M \times D}$ and $\mathcal{F} \subset\left(\mathbb{C}^{\mathbb{Z}}\right)^{M \times D}$ be the subspaces of allowed subband models, analysis and synthesis filterbank polyphase matrices, respectively. Then, the system approximation problem can be written as

$$
(\boldsymbol{\Gamma}, \mathbf{H}, \mathbf{F})=\underset{\tilde{\boldsymbol{\Gamma}} \in \mathcal{G}, \tilde{\mathbf{H}} \in \mathcal{H}, \tilde{\mathbf{F}} \in \mathcal{F}}{\arg \min }\left\|\mathbf{G}-\tilde{\mathbf{F}}^{*} * \tilde{\boldsymbol{\Gamma}} * \tilde{\mathbf{H}}\right\|_{r_{x}}
$$

\section{OPTIMIZATION ALGORITHM}

The problem (4) is a non-linear least-squares optimization problem, which we solve using the ALS method. More precisely, if we fix two of the elements in $(\boldsymbol{\Gamma}, \mathbf{H}, \mathbf{F})$, the optimization of the third element is a linear optimization problem which can be solved using linear least-squares (LLS). The
ALS algorithm cyclically repeats these three steps. Below we analyze each step separately. We define the following transformations:

If $\mathbf{X}$ is a vector in $\left(\mathbb{C}^{\mathbb{Z}}\right)^{B \times 1}$, and $m, n \in \mathbb{Z}$, then $\underline{\mathbf{X}}=$ seq2 $2 \operatorname{col}_{m, n}\{\mathbf{X}\}$ is given by $\underline{\mathbf{X}}=\left[\underline{\mathbf{X}_{1}^{T}}, \cdots, \mathbf{X}_{B}^{T}\right]^{T}$ with $\underline{\mathbf{X}_{i}}=$ $\left[[\mathbf{X}]_{i}(m), \cdots,[\mathbf{X}]_{i}(n)\right]^{T}$.

If $\mathbf{X}$ is a matrix in $\left(\mathbb{C}^{\mathbb{Z}}\right)^{B \times A}$, and $n \in \mathbb{Z}$, then $\underline{\mathbf{X}}=$ $\operatorname{convmat}_{n}\{\mathbf{X}\}$ denotes the matrix $\underline{\mathbf{X}}=\left[\underline{\mathbf{X}}_{b, a}\right]_{b, a=1,1}^{B, A}$, with each submatrix $\left[\underline{\mathbf{X}}_{b, a}\right]_{i, j}=\mathbf{X}(i-j)$ for all $i, j \in\{1, \cdots, n\}$.

If $\mathbf{X}, \mathbf{Y} \in\left(\mathbb{C}^{\mathbb{Z}}\right)^{B \times A}$, then $\mathbf{X} \odot \mathbf{Y} \in\left(\mathbb{C}^{\mathbb{Z}}\right)^{B \times A}$ denotes the entry-wise convolution, i.e., $[\mathbf{X} \odot \mathbf{Y}]_{b, a}=[\mathbf{X}]_{b, a} *[\mathbf{Y}]_{b, a}$.

Theorem 1 (Optimization of $\Gamma$ ) Let $\mathcal{G} \subset\left(\mathbb{C}^{\mathbb{Z}}\right)^{M \times M}$ be the subspace of diagonal matrix sequences with FIR filters of tap size $l_{\Gamma}$ and $d_{\Gamma}$ non-causal taps, on each diagonal entry, and let $m_{\boldsymbol{\Gamma}}=-d_{\boldsymbol{\Gamma}}$ and $n_{\boldsymbol{\Gamma}}=l_{\boldsymbol{\Gamma}}-d_{\boldsymbol{\Gamma}}-1$. Then, the solution of (4), for fixed $\mathbf{H}$ and $\mathbf{F}$, is given by

$$
\boldsymbol{\Gamma}=\operatorname{diag}\left\{\operatorname{seq} 2 \operatorname{col}_{m_{\boldsymbol{\Gamma}}, n_{\Gamma}}^{-1}\left\{\underline{\mathbf{M}}^{\dagger} \mathbf{v}\right\}\right\}
$$

where

$$
\begin{aligned}
\underline{\mathbf{M}} & =\operatorname{convmat}_{n_{\Gamma}-m_{\Gamma}+1}\left\{\phi_{0}^{D}\left(\mathbf{h} * r_{x} * \mathbf{h}^{*}\right) \odot \phi_{0}^{D}\left(\mathbf{f} * \mathbf{f}^{*}\right)\right\} \\
\underline{\mathbf{v}} & =\operatorname{seq}_{2 \operatorname{col}_{m_{\boldsymbol{\Gamma}}, n_{\Gamma}}}\left\{\operatorname{diag}^{-1}\left\{\phi_{0}^{D}\left(\mathbf{f} * g * r_{x} * \mathbf{h}^{*}\right)\right\}\right\}
\end{aligned}
$$

Theorem 2 (Optimization of $\mathbf{H}$ ) Let $\mathcal{H} \subset\left(\mathbb{C}^{\mathbb{Z}}\right)^{M \times D}$ be the subspace of polyphase representations of Gabor filterbanks whose filters are causal and FIR with tap sizes $l_{\mathbf{h}}$. The solution of (4), for fixed $\mathbf{\Gamma}$ and $\mathbf{F}$, is given by

$$
\mathbf{H}=\mathcal{W}_{M} \mathbf{L}_{2} \operatorname{diag}^{-1}\left\{\underline{\mathbf{M}}^{\dagger} \underline{\mathbf{v}}\right\} \mathbf{L}_{1}
$$

where

$$
\begin{aligned}
\underline{\mathbf{M}} & =\left(\mathbf{A} \odot \mathbf{C}^{T}\right)(0) \\
\mathbf{A} & =\mathbf{L}_{2}^{*} \mathcal{W}_{M}^{*} \mathbf{B} \mathcal{W}_{M} \mathbf{L}_{2} \\
\mathbf{B} & =\left(\operatorname{diag}^{-1}\left\{\boldsymbol{\Gamma}^{*}\right\} * \operatorname{diag}^{-1}(\boldsymbol{\Gamma})^{T}\right) \odot \phi_{0}^{D}\left(\mathbf{f} * \mathbf{f}^{*}\right) \\
\mathbf{C} & =\mathbf{L}_{1} * \mathbf{R}_{\mathbf{x}} * \mathbf{L}_{1}^{*} \\
\underline{\mathbf{v}} & =\operatorname{diag}^{-1}\left\{\mathbf{L}_{2}^{*} \mathcal{W}_{M}^{*} \boldsymbol{\Gamma}^{*} * \phi_{0}^{D}\left(\mathbf{f} * r_{x} * g\right) * \mathbf{L}_{1}^{*}\right\}(0)
\end{aligned}
$$

with $\mathbf{L}_{1}, \mathbf{L}_{2}$ and $\mathcal{W}_{M}$ defined as in Section 3.1.

Theorem 3 (Optimization of F) Let $\mathcal{F} \subset\left(\mathbb{C}^{\mathbb{Z}}\right)^{M \times D}$ be the subspace of polyphase representations of Gabor filterbanks whose filters are causal and FIR with tap sizes $l_{\mathbf{f}}$. The solution of (4), for fixed $\mathbf{\Gamma}$ and $\mathbf{H}$, is given by

$$
\mathbf{F}=\mathcal{W}_{M} \mathbf{L}_{2} \operatorname{diag}^{-1}\left\{\underline{\mathbf{M}}^{\dagger} \underline{\mathbf{v}}\right\} \mathbf{L}_{1}
$$

where

$$
\begin{aligned}
\underline{\mathbf{M}} & =\left(\mathbf{A} \odot \mathbf{C}^{T}\right)(0) \\
\mathbf{A} & =\mathbf{L}_{2}^{*} \mathcal{W}_{M}^{*} \mathbf{B} \mathcal{W}_{M} \mathbf{L}_{2} \\
\mathbf{B} & =\left(\operatorname{diag}^{-1}\{\boldsymbol{\Gamma}\} * \operatorname{diag}^{-1}\left(\boldsymbol{\Gamma}^{*}\right)^{T}\right) \odot \phi_{0}^{D}\left(\mathbf{h} * r_{x} * \mathbf{h}^{*}\right) \\
\mathbf{C} & =\mathbf{L}_{1} * \mathbf{L}_{1}^{*} \\
\underline{\mathbf{v}} & =\operatorname{diag}^{-1}\left\{\mathbf{L}_{2}^{*} \mathcal{W}_{M}^{*} \boldsymbol{\Gamma}^{*} * \phi_{0}^{D}\left(\mathbf{f} * r_{x} * g\right) * \mathbf{L}_{1}^{*}\right\}(0)
\end{aligned}
$$




\begin{tabular}{|c|c|c|c|c|}
\cline { 2 - 5 } \multicolumn{1}{c|}{} & \multicolumn{2}{c|}{ Azy $=0$, Ele $=0$} & \multicolumn{2}{c|}{ Azy $=45$, Ele $=40$} \\
\cline { 2 - 5 } \multicolumn{1}{c|}{} & Left & Right & Left & Right \\
\hline OS/A & $-37.27 \mathrm{~dB}$ & $-39.41 \mathrm{~dB}$ & $-40.47 \mathrm{~dB}$ & $-28.24 \mathrm{~dB}$ \\
SB & $-38.67 \mathrm{~dB}$ & $-38.22 \mathrm{~dB}$ & $-39.58 \mathrm{~dB}$ & $-30.23 \mathrm{~dB}$ \\
\hline
\end{tabular}

Table 2. Approximation errors for the OS/A and subband (SB) methods for two sound positions and both ears.

Initialization: The recursive method introduced above requires an initialization. To this end, we choose the analysis and synthesis filterbanks to be equal (i.e., $f_{0}=h_{0}$ ), and the filters $h_{m}, m \in\{1, \cdots, M\}$, to be FIR approximations (in a square sense) of ideal filterbanks whose frequency response satisfy: (a) its support is contained in an interval of measure $2 \pi / D$, and (b) the union of all $M$ supports cover the interval $[-\pi, \pi]$. An example is shown in Fig. 3. As pointed out in [2], this guarantees that the approximation error can be made arbitrarily small with a diagonal subband model of sufficiently large tap size.

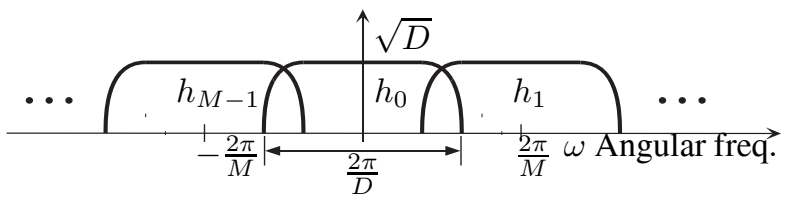

Fig. 3. Ideal filterbank frequency response.

\section{APPLICATION TO BINAURAL VIRTUAL ACOUSTICS}

The subband technique was used to optimize a binaural virtual acoustics application. We processed an HRTF set of a human subject which was measured at our facilities, using the multiple exponential sweep method [12]. The HRTFs are 512 tap filters (measured at $48 \mathrm{kHz}$ ) for the left and right ear. We compared the performance (in terms of $\mathrm{CC}$ and latency) of the subband technique with that of the OS/A methods for two representative sound positions: frontal (azimuth $=0^{\circ}$ and elevation $=0^{\circ}$ ) and lateral-elevated (azimuth $=45^{\circ}$ and elevation $=40^{\circ}$ ). In virtual acoustics, the localization accuracy does not significantly decreases when the HRIR are truncated to $5 \mathrm{~ms}$. (i.e., 240 taps) [13]. Hence, in order to compare both approaches, we choose $l_{g}=240$ for the OS/A methods, and we design the subband method to achieve an approximation error comparable to that introduced by the aforementioned truncation. To this end, we choose $M=7, D=5, l_{\mathbf{h}}=l_{\mathbf{f}}=15$, $l_{\Gamma}=52$ and $d_{\Gamma}=0$. The errors for both methods are shown in Table 2.

According to Table 1, with this subband design we achieve a delay of 14 taps (i.e., $0.3 \mathrm{~ms}$.) and a computational cost of 82 multiplications per sample. If the same delay were to be achieved with a OS/A method, we would require $L=15$, which would require 152 multiplications per sample. On the other hand, to achieve a computational cost of 82 multiplications per sample, we would require $L=30$, which would introduce a latency of 29 taps (i.e., $0.6 \mathrm{~ms}$.). Hence, we conclude that the performance of the subband method almost doubles that of the OS/A methods, i.e., it halves computations for the same latency or halves latency for the same computational cost.

\section{REFERENCES}

[1] Damián Marelli, "A functional analysis approach to subband system identification and approximation," IEEE Transactions on Signal Processing, vol. 55, no. 2, pp. 493-506, February 2007.

[2] Damián Marelli and Minyue Fu, "Performance analysis for subband identification," IEEE Transactions on Signal Processing, vol. 51, no. 12, pp. 3128-3142, December 2003.

[3] Youhong Lu and Joel Morris, "Gabor expansion for adaptive echo cancellation," IEEE Signal Processing Magazine, vol. 16, no. 2, pp. 68-80, March 1999.

[4] Damián Marelli and Minyue Fu, "A subband approach to channel estimation and equalization for dmt and ofdm systems," IEEE Transactions on Communications, vol. 53, no. 11, pp. 1850-1858, November 2005.

[5] Hans G. Feichtinger and Krzysztof Nowak, "A first survey of Gabor multipliers," in Advances in Gabor analysis, Appl. Numer. Harmon. Anal., pp. 99-128. Birkhäuser Boston, Boston, MA, 2003.

[6] P. Balazs, "Hilbert-Schmidt operators and frames - classification, best approximation by multipliers and algorithms," To appear in the International Journal of Wavelets, Multiresolution and Information Processing.

[7] Wim P. Krijnen, "Convergence of the sequence of parameters generated by alternating least squares algorithms," Comput. Statist. Data Anal., vol. 51, no. 2, pp. 481-489, 2006.

[8] John G. Proakis and Dimitris G. Manolakis, Digital signal processing: principles, algorithms, and applications, Prentice Hall International, Englewood Cliffs, NJ., third edition, 1996.

[9] F.L. Wightman and D.J. Kistler, "Headphone simulation of free-field listening. i. stimulus synthesis," Journal of the Acoustical Society of America, vol. 85, no. 2, pp. 858 - 67, 1989/02/.

[10] S. Weiss and R.W. Stewart, "Fast implementation of oversampled modulated filter banks," Electronics Letters, vol. 36, no. 17, pp. 1502-1503, August 2000.

[11] P.P. Vaidyanathan, Multirate Systems and Filterbanks, Prentice Hall, Englewood Cliffs, N.J., 1993.

[12] Piotr Majdak, Peter Balazs, and Bernhard Laback, "Multiple exponential sweep method for fast measurement of headrelated transfer functions," AES: Journal of the Audio Engineering Society, vol. 55, no. 7-8, pp. 623 - 636, 2007.

[13] Melis A. Senova, Ken I. McAnally, and Russel L. Martin, "Localization of virtual sound as a function of head-related impulse response duration," Journal of the Audio Engineering Society, vol. 50, no. 1, pp. 57-66, January 2002. 